\title{
EXPERIMENTAL AND THEORETICAL STUDY OF GAIN TEMPORAL PROFILES IN ELECTRON-BEAM PUMPED HIGH-PRESSURE Ar-Xe AND He-Ne-Kr LASERS
}

\author{
A.Yu. DUDIN, I. V. SEMENOVA, I.V. KHOLIN and D.A. ZAYARNYI \\ P.N. Lebedev Physical Institute, Leninsky Prospect 53, Moscow 117924, USSR
}

A series of dynamio parameter measurements of the highpressure eleotron-beam-ezcited atomic Ar-Xe laser and the $\mathrm{He}-\mathrm{Ne}-\mathrm{Kr}$ laser has been made. Temporal profiles of the smallsignal gain for $1.73,2.03,2.65,3.37,3.51 \mu \mathrm{m}$ Iines on the $5 d-6 p$ xenon atomic trasitions with Ar-Xe mixtures and for $0.585,0.703,0.725 \mu \mathrm{m}$ lines on the $3 \mathrm{p}-3 \mathrm{~s}$ neon atomic transition with He-Ne-Kr mixtures was obtained for various values of the mixture pressure and the e-beam pump rate.

The experimental setup "Tandem" consist of two e-beam lasers with an active volume 10 liters and the maximal mixture pressure $3.5 \mathrm{~atm}$ eaoh. The $350 \mathrm{keV}$ e-beam ourrent density incide the experimental laser chamber was approximately $1.7 \mathrm{~A} / \mathrm{cm}^{2}$ in $3.5 \mu \mathrm{s}$ pulse. The sourse of the input probe beam was the e-beam Ar-Xe or He-Ne-Kr laser with hot cathode and a $30 \mu s$ e-beam pulse. Typioal experimental amplitude small-signal gains lie in the range $0.25-0.1 \mathrm{om}^{-1}$ for the Ar-Xe laser and near $0.02 \mathrm{~cm}^{-1}$ for the He-Ne-Kr laser.

Based on the result of gain measurements and the conceptions of perfomances of these lasers $[1,2]$, authors report on computer models of the e-beam Ar-Xe laser and the He-Ne-Kr. laser weIl disorabed generation and gain parameters of this lasers. The author hypothesis that dissociative recombination of heterodimer ArXe ${ }^{+}$directly populates upper laser levels (5d manifold) of zenon and conventional constants of plasma chemical processes in the Ar-Xe laser allows to compute close to experimental laser output, laser spectral composition and efficienoy parameters. Also experimental unknown rate constants of the heavy-particle quenching low laser levels. (6p manifold) by argon were defined.

1. N.G.Basov at all.-IEEE J.Quant. Electron.,QE-21,1756 (1985).

2. N.G.Basov at all.-Sov. J. Quant. Fleotron., 15, 1004 (1985). 